\title{
Evaluación in vitro del efecto de Neisseria gonorrhoeae y los factores solubles producto de su metabolismo sobre la calidad espermática
}

\author{
Jenniffer Puerta Suárez, MSc ${ }^{1 a}$, Walter D. Cardona Maya, MSc, PhD. ${ }^{1 b}$ \\ ${ }^{1}$ Grupo Reproducción, Departamento de Microbiología y Parasitología, Facultad de Medicina, Sede de Investigación \\ Universitaria, Universidad de Antioquia, Medellín, Colombia.
}

\footnotetext{
a Microbióloga Bioanalista.

bacteriólogo.
}

\section{RESUMEN}

Antecedentes: N. gonorrhoeae es una de las principales causas de uretritis, prostatitis y epididimitis en hombres. En el tracto urogenital masculino, esta bacteria o los factores solubles producto de su metabolismo pueden interactuar con los espermatozoides y alterar su calidad. Objetivos: Determinar el efecto de la incubación in vitro de N. gonorrhoeae y los factores solubles producto de su metabolismo sobre la calidad espermática y evaluar la interacción bacteria-espermatozoide. Método: Se realizó incubación de una concentración 0,5 McFarland de N. gonorrhoeae y de los factores solubles producto de su metabolismo bacteriano con muestras de semen de voluntarios aparentemente sanos. Se cuantificaron los parámetros espermáticos convencionales (movilidad y viabilidad) y funcionales (potencial de membrana mitocondrial, integridad y lipoperoxidación de la membrana espermática, detección de especies reactivas del oxígeno, integridad de la cromatina y expresión de Anexina V), empleando microscopía y citometría de flujo, respectivamente. Para evaluar la interacción de N. gonorrhoeae con los espermatozoides humanos se realizaron extendidos en placas para su observación al microscopio. Resultados: N. gonorrhoeae puede unirse al espermatozoide y disminuir la viabilidad espermática luego de 1,5 horas de incubación con los espermatozoides humanos $(84,5 \%$ vs $66,5 \%, p<0,05)$, sin afectar los parámetros espermáticos funcionales. Conclusión: N. gonorrhoeae interactúa con los espermatozoides humanos afectando la viabilidad espermática.

\section{PALABRAS CLAVE: Neisseria gonorrhoeae, factores solubles bacterianos, calidad seminal, parámetros espermáticos convencionales y funcionales}

\section{SUMMARY}

Background: N. gonorrhoeae is a major cause of urethritis, prostatitis and epididymitis in men. In the male urogenital tract, the bacteria or soluble products of their metabolism may interact with sperm, and alter their quality. Aims: To determine the in vitro effect of incubation of N. gonorrhoeae and soluble products of their metabolism on sperm quality and assess the bacteria-sperm interaction. Methods: Soluble products of bacterial metabolism and 0.5 McFarland concentration of N. gonorrhoeae were incubated with semen samples from healthy volunteers. Conventional (motility and viability) and functional sperm parameters (potential of mitochondrial membrane integrity and sperm membrane lipid peroxidation, detection of reactive oxygen species, chromatin integrity and expression of annexin V) were quantified using microscopy and cytometry flow, respectively. To assess the interaction of $\mathrm{N}$. gonorrhoeae to human sperm microscopic observation was performed. Results: N. gonorrhoeae can join the sperm and decreases sperm viability after 1.5 hours of 
incubation with human sperm $(84.5 \%$ vs $66.5 \%, p<0.05)$, without affecting the functional sperm parameters. Conclusion: N. gonorrhoeae interacts with human sperm affecting sperm viability.

\section{KEY WORDS: Neisseria gonorrhoeae, soluble products, semen quality, conventional and functional sperm parameters}

\section{INTRODUCCIÓN}

N. gonorrhoeae es un diplococo gran negativo, aerobio no esporulado e inmóvil, implicado en diferentes infecciones del tracto genitourinario tanto masculino como femenino $(1,2)$. El gonococo, como también suele denominarse frecuentemente a esta bacteria, causa infecciones severas en las mujeres que incluyen la enfermedad pélvica inflamatoria (EPI), endometritis, infección de las trompas de Falopio, abscesos ováricos y peritonitis pélvica $(1,3)$ dejando secuelas a largo plazo como la infertilidad (4). La infección masculina trae como consecuencia la uretritis, prostatitis, epididimitis e infertilidad $(5,6)$. La relevancia de la infección gonocócica radica en su capacidad de potenciar otras infecciones de transmisión sexual como las originadas por el virus de la inmunodeficiencia humana $(\mathrm{VIH})(1)$.

El uso de antimicrobianos ha disminuido la incidencia de la infección gonocócica aunque sigue siendo común entre los jóvenes de países industrializados como Estados Unidos, donde el $75 \%$ de los casos reportados para el año 2005 ocurrieron en el grupo de edad de 15-29 años y la tasa más alta de incidencia se presentó entre los 15-19 años con 625 casos por cada 100.00 habitantes (3).

Desde hace más de 30 años se viene investigando los mecanismos de unión entre los espermatozoides con esta bacteria y su capacidad de transporte desde el tracto genital masculino hasta el femenino $(7,8)$ y esa misma interacción puede estar relacionada con afectar la fertilidad masculina, por el daño en la calidad seminal. N. gonorrhoeae se une a las células de su único hospedero, el humano, a través del contacto directo con las mucosas empleando la molécula $\operatorname{CD} 46(7,9)$ y estructuras de superficie como el pili $(3,10)$. El pili bacteriano causa hemoaglutinación por la adherencia a los eritrocitos (11) y esta estructura puede ser empleada para unirse a la célula espermática, aunque no es la única; las proteínas de la membrana externa y los lipo-oligosacáridos parecen desempeñar un papel crítico en la unión del microorganismo a las células de la mucosa genitourinaria (1).

Adicionalmente, el efecto que puedan tener las infecciones genitourinarias masculinas sobre la calidad seminal puede estar relacionado no solo con la presencia de la bacteria en el tracto reproductivo masculino, los microorganismos durante su metabolismo pueden secretar sustancias al medio que podrían incidir en la calidad seminal, como se ha reportado con la bacteria uropatógena E. coli (12).

El objetivo de este trabajo fue determinar el efecto de la incubación in vitro de N. gonorrhoeae y los factores solubles producto de su metabolismo sobre la calidad espermática y adicionalmente evaluar la interacción entre la bacteria con los espermatozoides humanos.

\section{MATERIALES Y MÉTODOS}

Muestras de semen: Se emplearon 12 muestras de semen de voluntarios aparentemente sanos, mayores de edad, obtenidas por masturbación y colectadas en un recipiente estéril luego de una abstinencia sexual de 2 a 5 días y con parámetros seminales iguales o superiores a los límites inferiores de referencia establecidos por la Organización Mundial de la Salud (OMS) (13). La concentración espermática se determinó empleando la cámara de Makler (Sefi-Medical Instruments, Israel) (14).

Obtención de los factores solubles: Se empleó la cepa bacteriana N. gonorrhoeae ATCC 43063, y se cultivó en agar Thayer Martin (ICMT, Medellín, Colombia) a $37^{\circ} \mathrm{C} / 5 \% \mathrm{CO} 2$ y los factores solubles producto del metabolismo bacteriano se obtuvieron incubando durante 30 minutos un inóculo bacteriano a una concentración de 0,5 McFarland en PBS (Gibco®, NY, USA), posteriormente, el medio se centrifugó a $600 \mathrm{~g} / 10$ minutos, se recolectó el sobrenadante y se filtró con una membrana bacteriológica de 0,2 $\mu \mathrm{m}$ (Advantec Industries, China), se alicuotó y se almacenó a $-20^{\circ} \mathrm{C}$ hasta su uso.

Evaluación de la interacción entre $N$. gonorrhoea y los espermatozoides humanos. Con el fin de evaluar la interacción entre la bacteria y los espermatozoides humanos se incubaron $5 \times 10^{6}$ espermatozoides móviles seleccionados empleando la técnica Swim-up con una concentración bacteriana de $0,5 \mathrm{McF}$ arland por dos horas a $37^{\circ} \mathrm{C}$, posteriormente se realizaron 5 lavados centrifugando a $300 \mathrm{~g} / 5 \mathrm{~min}$, cambiando de vial en los lavados 1, 3 y 5 . De la mezcla final de bacterias y espermatozoides se tomaron $20 \mu \mathrm{L}$ y se realizaron extendidos en placas porta objetos para evaluar la presencia de diplococos intra y extracelulares mediante la tinción 
de Gram y con el colorante STAT III (Origio, Dinamarca) realizando observaciones en un microscopio Nikon Eclipse E200 (Nikon, Tokyo, Japón) con un objetivo de $100 \mathrm{X}$.

Evaluación de N. gonorrhoeae y sus factores solubles sobre los parámetros espermáticos convencionales. Inicialmente se preparó un inóculo bacteriano de $N$. gonorrhoeae a una concentración 0,5 McFarland en PBS y se atemperó una alícuota de los factores solubles previamente preparados. Las muestras de semen fueron incubadas con la bacteria o sus factores solubles en una proporción 1:1 (100 $\mu \mathrm{L}$ de semen y $100 \mu \mathrm{L}$ de inóculo bacteriano/factores solubles) durante 2 horas a $37^{\circ} \mathrm{C}$. El efecto sobre la movilidad y la viabilidad espermática se determinó inmediatamente luego de la incubación de las muestras de semen con el inóculo bacteriano o sus factores solubles y cada $30 \mathrm{mi}-$ nutos hasta completar las 2 horas de incubación. Los resultados son expresados como la mediana y el rango del porcentaje de movilidad tipo I+II y de viabilidad.

Evaluación de N. gonorrhoeae y sus factores solubles sobre los parámetros espermáticos funcionales. La evaluación de los parámetros espermáticos funcionales se realizó por citometría de flujo (CoulterEpics XL - BeckmanCoulter, CA, USA) analizando entre 5.000 y 10.000 espermatozoides de acuerdo a los protocolos previamente estandarizado en el Grupo Reproducción (15-19) y luego de incubar 250 $\mu \mathrm{L}$ de semen con el inóculo bacteriano o con los factores solubles a $37^{\circ} \mathrm{C} / 2$ horas en proporción 1 a 1 . Posterior a la incubación, se obtuvieron alícuotas de
$2 \times 10^{6}$ y de $5 \times 10^{6}$ espermatozoides para ser incubadas con diferentes fluorocromos (Tabla I) de acuerdo al parámetro a evaluar (15,16,20-22).

Análisis estadístico. Los resultados de las citometrías de flujo fueron graficados y procesados utilizando el programa FlowJo 7.6 (TreeStar, Inc. Oregon, USA). Todos los resultados obtenidos se expresaron como la mediana y el rango. Para comparar el efecto de los factores solubles o las bacterias sobre los parámetros espermáticos se realizó una prueba ANOVA no paramétrica (Friedman) y el pos-test de Dunns considerando significancia estadística un $p<0,05$ utilizando el programa GraphPadPrism 6 (Graphpad, CA, USA).

\section{RESULTADOS}

Inicialmente, se observó que las bacterias pueden unirse a los espermatozoides humanos en sus tres estructuras básicas: cabeza, pieza intermedia y cola (Figura 1); luego de la incubación de N. gonorrhoeae y de los factores solubles producto de su metabolismo con los espermatozoides humanos, no se observan diferencias estadísticamente significativas en la movilidad espermática, ni cambios en la muestra control con respecto a este parámetro. En cuanto a la viabilidad, la bacteria disminuye en un $21,3 \%$ este parámetro luego de una hora y 30 minutos de incubación con respecto al control de viabilidad inicial $(84,5 \%$ vs $66,5 \%, p<0,05)$. No se observaron cambios estadísticamente significativos en cuanto a la movilidad inicial de la muestra control y luego de 2 horas de incubación (Figura 2).

\section{Tabla I \\ PRUEBAS FUNCIONALES ESPERMÁTICAS Y SUS FLUOROCROMOS}

\begin{tabular}{|c|c|c|}
\hline Prueba & Reactivos para la tinción & Referencias \\
\hline Potencial de membrana mitocondrial & $\begin{array}{l}\text { loduro de propidio (IP, } 0.25 \mathrm{mg} / \mathrm{mL} \text {, Molecular Pro- } \\
\text { bes } ₫ \text { Inc, OR, USA) y } 3,3 \text { 'dihexiloxacarbocianina } \\
\text { (DIOC6, } 10 \mathrm{nM} \text { Molecular Probes } ₫ \text { ). }\end{array}$ & $(15,16)$ \\
\hline Integridad de la membrana plasmática & $\begin{array}{l}\text { IP (concentración final } 0.25 \mathrm{mg} / \mathrm{mL} \text { ) y Sybr } 14 \text { (LIVE/ } \\
\text { DEAD® Sperm Viability Kit, Molecular Probes }{ }^{\circledR} \\
1 \mu \mathrm{M}) .\end{array}$ & $(15,16)$ \\
\hline Lipoperoxidación de la membrana & $\begin{array}{l}\text { 4,4-difluoro-4-bora-3a-4a-diaza-s-indaceno (BODI- } \\
\text { PY C11 Molecular Probes } ® \text { Inc, OR, USA). }\end{array}$ & $(15,20)$ \\
\hline Especies reactivas del oxígeno & $\begin{array}{l}\text { Di-acetato de 2',7' diclorofluoresceína (DCFH-DA, } 1 \\
\mu \mathrm{M} \text { Sigma-Aldrich, St Louis, MO, USA) y IP }(0.25 \mathrm{mg} / \\
\mathrm{mL}) \text {. }\end{array}$ & $(15,16)$ \\
\hline Expresión de fosfatidil serina & $\begin{array}{l}\text { Anexina V FITC/IP, con Anexina V FITC }(0,5 \mathrm{mg} / \mathrm{mL}, \mathrm{E} \\
\text { Biosciencie, San Diego, CA, USA). }\end{array}$ & $(21)$ \\
\hline Fragmentación del ADN & $\begin{array}{l}\text { Naranja de acridina (NA, } 0.006 \mathrm{mg} / \mathrm{mL} \text { Sigma-Al- } \\
\text { drich, St Louis, MO, USA). }\end{array}$ & $(15,16,22)$ \\
\hline
\end{tabular}




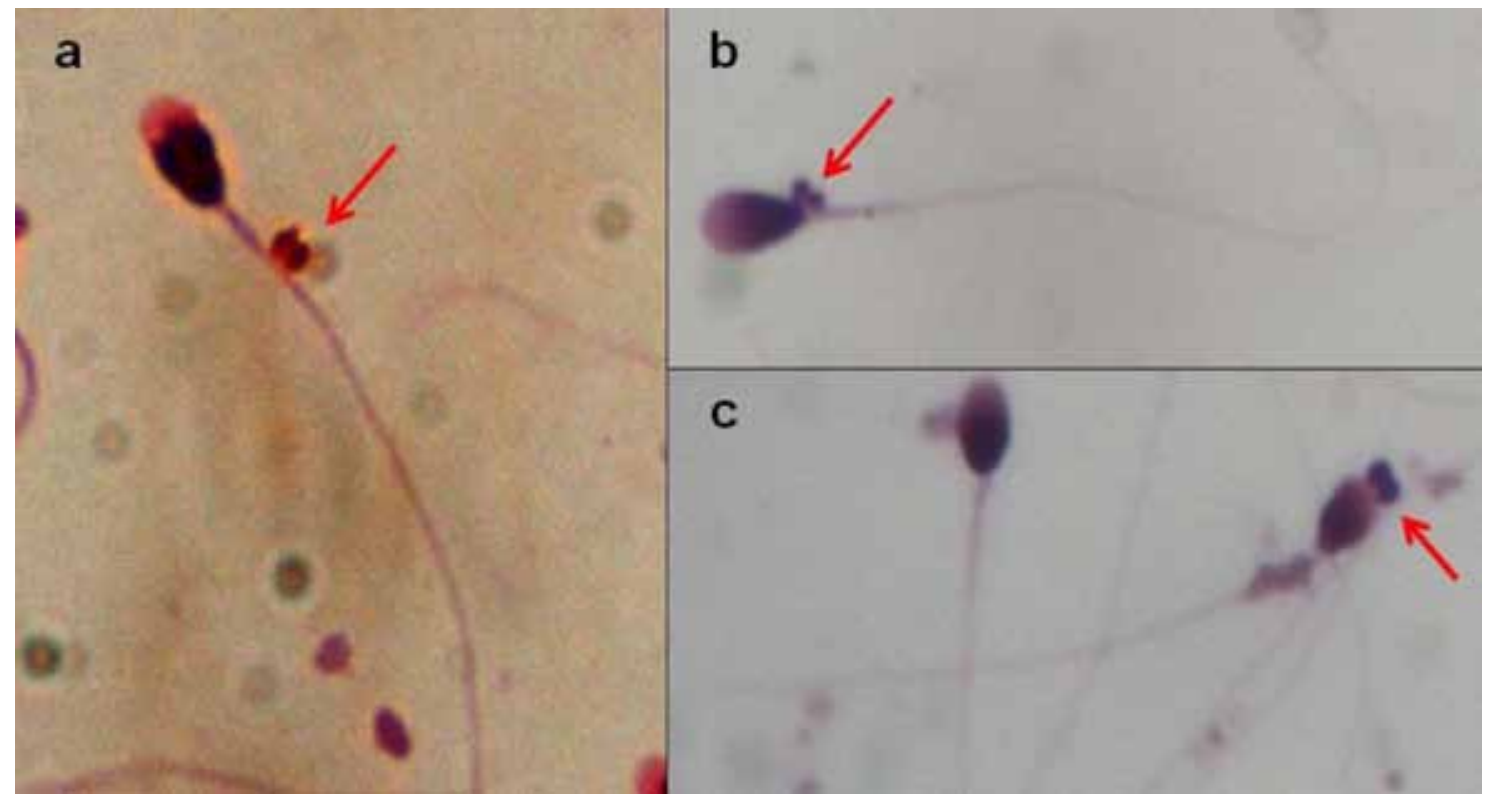

Figura 1. Interacción entre el espermatozoide humano con N. gonorrhoeae. La bacteria puede unirse a la cola (a), la pieza intermedia (b) o la cabeza (c). Observaciones realizadas con la tinción de Gram (a) y con el colorante de andrología STAT II (b y c).

a

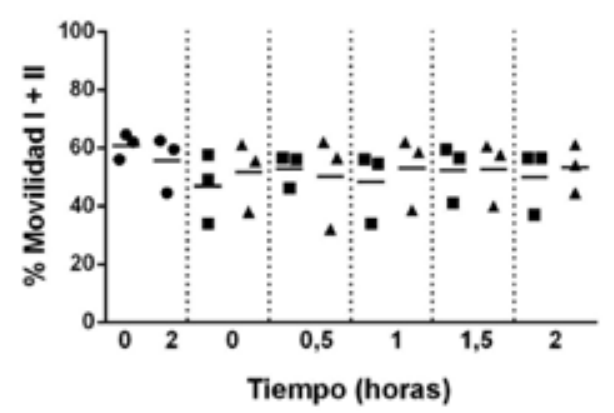

b

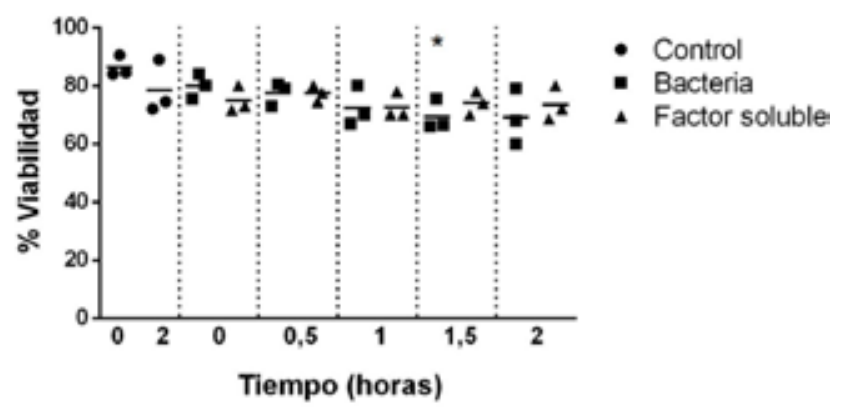

Figura 2. Efecto de la incubación in vitro de $N$. gonorrhoeae y los factores solubles producto de su metabolismo sobre los parámetros espermáticos convencionales: a. Porcentaje de espermatozoides móviles (tipo I + II) y b. viabilidad espermática luego de la incubación in vitro de la bacteria y sus factores solubles con los espermatozoides humanos. Los datos son la mediana y el rango de 3 experimentos $\left({ }^{*} p<0,05\right)$. $N$. gonorrhoeae disminuye de forma estadísticamente significativa la viabilidad espermática con respecto al control luego de 1,5 horas de incubación.

Al incubar los espermatozoides humanos con $N$. gonorrhoeae o con los factores solubles producto de su metabolismo, no se observan cambios estadísticamente significativos en los parámetros espermáticos funcionales (Tabla II); sin embargo, se observa 2 veces más porcentaje de células con membrana celular desintegrada (positivas para SYBR y IP), una disminución del $7,9 \%$ en la producción de ERO y un aumento del $19,8 \%$ en el porcentaje de células con lipoperoxidación de la membrana en los espermatozoides incubados con la bacteria con respecto a la muestra control. 
Tabla II

EFECTO DE LA INCUBACIÓN IN VITRO DE N. GONORRHOEAEY LOS FACTORES SOLUBLES PRODUCTO DE SU METABOLISMO SOBRE LOS PARÁMETROS ESPERMÁTICOS FUNCIONALES

\begin{tabular}{|c|c|c|c|c|}
\hline Parámetro & Control & N. gonorrhoeae & Control & $\begin{array}{c}\text { Factores } \\
\text { solubles de } \\
\text { N. gonorrhoeae }\end{array}$ \\
\hline $\begin{array}{l}\text { Potencial de membrana mitocon- } \\
\text { drial alto (\%) }\end{array}$ & $\begin{array}{c}66,5 \\
(20,4-74,8)\end{array}$ & $\begin{array}{c}62,0 \\
(22,3-71,7)\end{array}$ & $\begin{array}{c}66,5 \\
(43,9-73,4)\end{array}$ & $\begin{array}{c}66,4 \\
(51,5-71,5)\end{array}$ \\
\hline Células con PMM Bajo (\%) & $\begin{array}{c}6,1 \\
(4,6-7,2)\end{array}$ & $\begin{array}{c}6,4 \\
(3,4-9,8)\end{array}$ & $\begin{array}{c}4,6 \\
(3,9-15,2)\end{array}$ & $\begin{array}{c}5,8 \\
(2,3-10,8)\end{array}$ \\
\hline Integridad de membrana (\%) & $\begin{array}{c}56,9 \\
(46,0-65,3)\end{array}$ & $\begin{array}{c}57,2 \\
(46,9-59,7)\end{array}$ & $\begin{array}{c}65,3 \\
(47,9-71,8)\end{array}$ & $\begin{array}{c}57,2 \\
(52,4-65,5)\end{array}$ \\
\hline Células moribundas (\%) & $\begin{array}{c}2,2 \\
(2,2-4,6)\end{array}$ & $\begin{array}{c}6,6 \\
(1,7-8,3)\end{array}$ & $\begin{array}{c}4,6 \\
(2,4-4,7)\end{array}$ & $\begin{array}{c}2,8 \\
(1,1-4,2)\end{array}$ \\
\hline Células necróticas (\%) & $\begin{array}{c}42,0 \\
(27,4-73,0)\end{array}$ & $\begin{array}{c}37,8 \\
(29,7-72,4)\end{array}$ & $\begin{array}{c}27,4 \\
(24,8-43,2)\end{array}$ & $\begin{array}{c}31,3 \\
(25,2-39,0)\end{array}$ \\
\hline Células DCF positivas (\%) & $\begin{array}{c}61,9 \\
(49,3-75,0)\end{array}$ & $\begin{array}{c}51,0 \\
(45,7-69,7)\end{array}$ & $\begin{array}{c}75,0 \\
(53,2-78,5)\end{array}$ & $\begin{array}{c}71,3 \\
(58,3-74,6)\end{array}$ \\
\hline Producción de EROs, IMF & $\begin{array}{c}6,3 \\
(1,7-6,6)\end{array}$ & $\begin{array}{c}5,8 \\
(1,9-7,4)\end{array}$ & $\begin{array}{c}6,3 \\
(2,2-6,5)\end{array}$ & $\begin{array}{c}7,7 \\
(1,5-8,4)\end{array}$ \\
\hline $\begin{array}{l}\text { Células con lipoperoxidación de } \\
\text { membrana (\%) }\end{array}$ & $\begin{array}{c}9,6 \\
(7,0-12,6)\end{array}$ & $\begin{array}{c}11,5 \\
(7,2-13,9)\end{array}$ & $\begin{array}{c}6,0 \\
(5,7-8,6)\end{array}$ & $\begin{array}{c}7,7 \\
(5,8-8,0)\end{array}$ \\
\hline Índice de fragmentación del ADN & $\begin{array}{c}8,8 \\
(3,5-14,0)\end{array}$ & $\begin{array}{c}8,9 \\
(4,7-11,9)\end{array}$ & $\begin{array}{c}6,4 \\
(3,6-14)\end{array}$ & $\begin{array}{c}9,8 \\
(5,1-12,9)\end{array}$ \\
\hline Células Anexina V positivas (\%) & $\begin{array}{c}8,9 \\
(7,5-12,3)\end{array}$ & $\begin{array}{c}8,4 \\
(6,1-8,9)\end{array}$ & $\begin{array}{c}8,9 \\
(7,5-12,3)\end{array}$ & $\begin{array}{c}8,8 \\
(6,0-9,1)\end{array}$ \\
\hline
\end{tabular}

De otro lado, en los espermatozoides incubados con los factores solubles producto del metabolismo bacteriano de $N$. gonorrhoeae, se observa un aumento del $26 \%$ en el porcentaje de espermatozoides con potencial de membrana mitocondrial bajo, un incremento del $53 \%$ del índice de fragmentación del ADN y una disminución del $12,4 \%$ de células con integridad de membrana y del $39 \%$ de las células con respecto a la muestra control.

\section{DISCUSIÓN}

N. gonorrhoeae es un diplococo gramnegativo responsable de la ITS conocida como gonorrea, la cual es altamente prevalente a nivel global $(2,11)$. Al evaluar la calidad seminal a través de los parámetros espermáticos luego de la incubación in vitro de la bacteria y los factores solubles producto de su metabolismo con los espermatozoides, observamos que N. gonorrhoeae disminuye de forma estadísticamente significativa la viabilidad espermática sin afectar la movilidad.
Adicionalmente, tanto la bacteria como los factores solubles producto de su metabolismo, pueden generar alteraciones en los parámetros espermáticos funcionales, similar a lo reportado en alteraciones como la prostatitis donde se incluye además, la disminución en la movilidad y la integridad del ADN espermático (23).

Aunque demostramos la interacción entre la célula espermática y la bacteria a través de sus tres componentes: cabeza, pieza intermedia y cola, técnicas de microscopía como la electrónica de transferencia serían de más utilidad por su capacidad de resolución mayor $(0,4$ a $0,8 \mathrm{~nm})$ en comparación con la microscopía convencional; por ejemplo Gómez y cols (11), evaluaron la interacción de N. gonorrhoeae empleando esta técnica y observaron que la bacteria se une a los espermatozoides empleando uno o varios pilis y que estas estructuras son susceptibles al tratamiento con tripsina a una concentración de $1 \mathrm{mg} / \mathrm{mL}$, lo que disminuye la adhesión. Adicionalmente, la interacción que observamos luego de 2 horas de incubación entre 
los espermatozoides y las bacterias, puede requerir menos tiempo, hay reportes de interacciones más tempranas en donde en 15 minutos de incubación se observa una adherencia del $50 \%$ del gonococo a las células espermáticas (8).

El pili es una estructura importante para la unión del gonococo con las células eucariotas en general, pero no es una estructura única (11), la unión con la célula espermática parece involucrar otros factores, debido a que las bacterias que no tienen pili se pueden unir al espermatozoide, pero lo hacen con mayor frecuencia aquellas que poseen la estructura $(8,11)$; su pérdida disminuye hasta en un $18 \%$ la adherencia del gonococo al espermatozoide y la presencia de hierro que favorece el desarrollo del pili, incrementa en un $4 \%$ su unión a la célula espermática (11). Por tanto, la unión bacteria/espermatozoide es frecuente y no es una característica única del gonococo, otras bacterias que presentan pili como E. coli pueden unirse a los espermatozoides humanos hasta en el $40 \%$ de los casos (8), incluso pueden unirse a virus como el VIH (24). Aún queda por definir si la unión entre ambas células es una propiedad de la bacteria o del espermatozoide, o es el resultado de la manipulación de uno o ambos (8).

En concordancia con nuestros resultados, Liu y cols (6) reportan que el gonococo no disminuye la movilidad espermática, pese a que la infección por este microorganismos es una causa importante de infertilidad masculina; sin embargo, lo más importante de la infección es que la gonorrea en su fase sintomática favorece la secreción de un alto número de bacterias en el semen lo que explica la eficacia de la transmisión gonocócica de los hombres a sus parejas $(1,7)$.

\section{CONCLUSIÓN}

A pesar de la controversia sobre el tema, las infecciones del tracto urogenital masculino causadas por N. gonorrhoeae afectan la calidad seminal, disminuyendo la viabilidad espermática, por lo que disminuye la probabilidad de alcanzar el éxito reproductivo.

Agradecimientos: Este trabajo fue financiado por Colciencias (111556933373) y por la Estrategia de Sostenibilidad, Grupo Reproducción, de la Universidad de Antioquia. JPS fue Joven Investigadora de Colciencias. Los investigadores agradecen a Jose Manuel Mayorga, Paula Lalinde, Lina Cadavid y Mariluz Giraldo por la ayuda técnica.

\section{REFERENCIAS}

1. Isbey SF, Alcorn TM, Davis RH, Haizlip J, Leone PA, Cohen MS. Characterisation of Neisseria gonorrhoeae in semen during urethral infection in men. Genitourin Med. 1997;73(5):378-82.
2. Wing JB, Jack DL, Lee ME, Pacey AA, Kinghorn GR, Read RC. Mannose-binding lectin is present in human semen and modulates cellular adhesion of Neisseria gonorrhoeae in vitro. Clin Exp Immunol. 2009;157(3):408-14.

3. Holmes K, Stamm W, Piot P, Wasserheit J, Corey L, Cohen M. Sexually Transmitted Diseases 4th ed. New York: McGraw. 2008.

4. Pellati D, Mylonakis I, Bertoloni G, Fiore C, Andrisani A, Ambrosini $G$, et al. Genital tract infections and infertility. Eur J Obstet Gynecol Reprod Biol. 2008;140(1):3-11.

5. Deguchi T, Yoshida T, Miyazawa T, Yasuda M, Tamaki $\mathrm{M}$, Ishiko $\mathrm{H}$, et al. Association of Ureaplasma urealyticum (biovar 2) with nongonococcal urethritis. Sexually transmitted diseases. 2004;31(3):192-5.

6. Liu JH, Li HY, Cao ZG, Duan YF, Li Y, Ye ZQ. Influence of several uropathogenic microorganisms on human sperm motility parameters in vitro. Asian journal of andrology. 2002;4(3):179-82.

7. Harvey HA, Porat N, Campbell CA, Jennings M, Gibson BW, Phillips NJ, et al. Gonococcal lipooligosaccharide is a ligand for the asialoglycoprotein receptor on human sperm. Mol Microbiol. 2000;36(5):1059-70.

8. James-Holmquest AN, Swanson J, Buchanan TM, Wende RD, Williams RP. Differential attachment by piliated and nonpiliated Neisseria gonorrhoeae to human sperm. Infection and immunity. 1974;9(5):897902.

9. Kirchner M, Heuer D, Meyer TF. CD46-independent binding of neisserial type IV pili and the major pilus adhesin, PilC, to human epithelial cells. Infection and immunity. 2005;73(5):3072-82.

10. Buchanan TM. Antigenic heterogeneity of gonococcal pili. The Journal of experimental medicine. 1975;141(6):1470-5.

11. Gomez Cl, Stenback WA, James AN, Criswell BS, Williams RP. Attachment of Neisseria gonorrhoeae to human sperm. Microscopical study of trypsin and iron. Br J Vener Dis. 1979;55(4):245-55.

12. Schulz M, Sanchez R, Soto L, Risopatron J, Villegas J. Effect of Escherichia coli and its soluble factors on mitochondrial membrane potential, phosphatidylserine translocation, viability, and motility of human spermatozoa. Fertil Steril. 2010;94(2):619-23.

13. WHO. Laboratory manual for the examination and processing of human semen. 2010.

14. Cardona-Maya W, Berdugo J, Cadavid A. Comparación de la concentración espermática usando la cámara de Makler y la cámara de Neubauer. Actas Urológicas Españolas. 2008;32(4):443-5.

15. Mayorga-Torres BJ, Camargo M, Agarwal A, du Plessis SS, Cadavid ÁP, Maya WDC. Influence of ejaculation frequency on seminal parameters. Reproductive Biology and Endocrinology. 2015;13(1):47.

16. Mayorga-Torres BJ, Cardona-Maya W, Cadavid A, Camargo M. Evaluación de los parámetros funcionales espermáticos en individuos infértiles normozooespermicos. Actas Urológicas Españolas. 2013;37(4):221-7.

17. Gil-Villa AM, Cardona-Maya W, Agarwal A, Sharma $R$, Cadavid $A$. Role of male factor in early recurrent embryo loss: do antioxidants have any effect? Fertil Steril. 2009;92(2):565-71.

18. Gil-Villa AM, Cardona-Maya W, Agarwal A, Sharma $\mathrm{R}$, Cadavid A. Assessment of sperm factors possibly 
involved in early recurrent pregnancy loss. Fertil Steril. 2010;94(4):1465-72.

19. Rodríguez E, Gil-Villa AM, Aguirre-Acevedo DC, Cardona-Maya W, Cadavid ÁP. Evaluación de parámetros seminales no convencionales en individuos cuyas parejas presentan muerte embrionaria temprana recurrente: en busca de un valor de referencia. Biomedica. 2011;31:100-7.

20. Aitken RJ, Wingate JK, De luliis GN, McLaughlin EA. Analysis of lipid peroxidation in human spermatozoa using BODIPY C11. Molecular Human Reproduction. 2007;13(4):203-11.

21. Glander HJ, Schaller J. Binding of annexin V to plasma membranes of human spermatozoa: a rapid assay for detection of membrane changes after cryostorage. Molecular human reproduction. 1999;5(2):109-15.
22. Evenson D, Jost L, Marshall D, Zinaman M, Clegg E, Purvis K, et al. Utility of the sperm chromatin structure assay as a diagnostic and prognostic tool in the human fertility clinic. Human Reproduction. 1999;14(4):1039.

23. Borovkova $N$, Korrovits $P$, Ausmees $K$, Türk $S$, Jõers $\mathrm{K}$, Punab M, et al. Influence of sexual intercourse on genital tract microbiota in infertile couples. Anaerobe. 2011;17(6):414-8.

24. Cardona-Maya W, Velilla PA, Montoya CJ, Cadavid A, Rugeles MT. In vitro human immunodeficiency virus and sperm cell interaction mediated by the mannose receptor. Journal of Reproductive Immunology. 2011;92(1):1-7. 